\title{
Polymyalgie rhumatismale
}

\author{
Hans Rosenberg MD, Samantha Halman MDM Méd, Krishan Yadav MD MSc
}

Citation : CMAJ 2021 November 22;193:E1770. doi : 10.1503/cmaj.210541-f

Voir la version anglaise de l'article ici : www.cmaj.ca/lookup/doi/10.1503/cmaj.210541

1 La polymyalgie rhumatismale (ou pseudopolyarthrite rhizomélique) est une maladie inflammatoire courante qui affecte les patients âgés

La polymyalgie rhumatismale arrive au second rang des maladies rhumatismales systémiques les plus courantes chez l'adulte. Le risque d'en souffrir au cours de la vie est de $2,4 \%$ chez les femmes et de $1,7 \%$ chez les hommes, et son incidence augmente entre les âges de 50 et 80 ans $^{1}$.

\section{Il est crucial de procéder à une anamnèse approfondie, car les manifestations \\ 2 physiques peuvent être subtiles}

La polymyalgie rhumatismale est caractérisée par une douleur bilatérale aux épaules d'apparition subite, accompagnée de raideur matinale. Plus rarement, le cou, la ceinture pelvienne et la partie proximale des cuisses sont affectés. Les symptômes constitutionnels sont fréquents; toutefois, le cas échéant, la fièvre est en général faible. L'amplitude des mouvements articulaires actifs est parfois restreinte, sans inflammation ni faiblesse articulaires observables².

Les critères de l'EULAR (European League Against Rheumatism) et de l'ACR (American

College of Rheumatology) permettent de distinguer la polymyalgie rhumatismale d'autres maladies qui lui ressemblent

Le diagnostic est probable chez les patients qui présentent les symptômes mentionnés plus haut, accompagnés d'une augmentation de la vitesse de sédimentation des érythrocytes (VSÉ) ou du taux de protéine $C$ réactive $(P C R)$ ou des deux, et qui répondent à la corticothérapie. Les critères de classification de l'EULAR et de l'ACR sont les plus sensibles et spécifiques et permettent de distinguer la polymyalgie rhumatismale d'autres maladies, telles que la polyarthrite rhumatoïde, l'arthrose de l'épaule et les myopathies ${ }^{3}$.

4

Le traitement repose sur de la prednisone pendant une période prolongée, débutée à

raison de 12,5-25 mg/j par voie orale

On observe généralement une amélioration en 24-72 heures, mais moins de la moitié des patients se rétablissent entièrement en 3 semaines $^{2}$. Il faut utiliser la dose de prednisone efficace la plus faible, habituellement pendant au moins 12 mois. Le sevrage à moins de $10 \mathrm{mg} / \mathrm{j}$ commencera une fois la rémission clinique et biochimique (normalisation de la VSÉ ou du taux de PCR ou des deux) obtenue ${ }^{4}$. On orientera le patient vers un spécialiste en présence de symptômes atypiques, d'effets indésirables du traitement, de symptômes réfractaires, de rechutes ou si l'on soupçonne une artérite à cellules géantes (maladie de Horton) ${ }^{5}$.

Chez tous les patients atteints de polymyalgie rhumatismale, il faut être à l'affût des signes et symptômes d'artérite à cellules géantes

L'artérite à cellules géantes accompagne $10 \%-21 \%$ des cas de polymyalgie rhumatismale ${ }^{1}$. Ses caractéristiques incluent céphalées, troubles visuels, claudication intermittente massétérine et sensibilité de l'artère temporale. Parmi les complications, mentionnons la cécité. Chez les patients qui présentent ces symptômes, il faut commencer la prednisone à une dose plus élevée, soit 40-60 mg/j, et demander rapidement une biopsie de l'artère temporale. 


\section{Références}

1. Buttgereit F, Dejaco C, Matteson EL, et al. polymyalgia rheumatica and giant cell arteritis: a systematic review. JAMA 2016;315:2442-58.

2. González-Gay MA, Matteson EL, Castañeda S. Polymyalgia rheumatica. Lancet 2017;390:1700-12.

3. Macchioni P, Boiardi L, Catanoso M, et al. Performance of the new 2012 EULAR/ACR classification criteria for polymyalgia rheumatica: comparison with the previous criteria in a single-centre study. Ann Rheum Dis 2014;73:1190-3.

4. Dejaco C, Duftner C, Cimmino MA, et al.; International Work Group for PMR and GCA. Definition of remission and relapse in polymyalgia rheumatica: data from a literature search compared with a Delphi-based expert consensus. Ann Rheum Dis 2011;70:447-53.

5. Dejaco C, Singh YP, Perel P, et al. 2015 Recommendations for the management of polymyalgia rheumatica: a European League Against Rheumatism/American College of Rheumatology collaborative initiative. Ann Rheum Dis 2015;74:1799-807.

Intérêts concurrents : Samantha Halman signale avoir reçu des subventions du Conseil médical du Canada, du Collège royal des médecins et chirurgiens du Canada, du Département de médecine de l'Hôpital d'Ottawa, du Département d'innovation en éducation médicale de l'Université d'Ottawa et de la Société canadienne de médecine interne, indépendamment des travaux soumis. Hans Rosenberg signale avoir reçu des honoraires de Podcast CAMP. Aucun autre intérêt concurrent n'a été déclaré.

Cet article a été révisé par des pairs.

Affiliations : Départements de médecine d'urgence (Rosenberg, Yadav) et de médecine (Halman), Université d'Ottawa et Hôpital d'Ottawa, Ottawa, Ont.

Propriété intellectuelle du contenu : Il s'agit d'un article en libre accès distribué conformément aux modalités de la licence Creative Commons Attribution (CC BY-NC-ND 4.0), qui permet l'utilisation, la diffusion et la reproduction de tout médium à la condition que la publication originale soit adéquatement citée, que l'utilisation se fasse à des fins non commerciales (c.-à-d., recherche ou éducation) et qu'aucune modification ni adaptation n'y soit apportée. Voir : https://creativecommons.org/licenses/by-nc-nd/4.0/deed.fr.

Correspondance : Hans Rosenberg, hrosenberg@toh.ca

Le $J A M C$ vous invite à soumettre vos textes pour la rubrique "Cinq choses à savoir ... » en ligne à http://mc.manuscriptcentral.com/cmaj. 